\title{
Rivaroxaban for the treatment of venous thromboembolism in patients with nephrotic syndrome and low AT-III: A pilot study
}

\author{
LIHUA ZHANG, HAITAO ZHANG, JIONG ZHANG, HONG TIAN, JU LIANG and ZHIHONG LIU \\ National Clinical Research Center of Kidney Disease, Jinling Hospital, \\ Nanjing University School of Medicine, Nanjing, Jiangsu 210016, P.R. China
}

Received May 11, 2017; Accepted September 22, 2017

DOI: $10.3892 /$ etm.2017.5471

\begin{abstract}
The anticoagulation effect of heparin requires adequate serum antithrombin (AT)-III levels. Rivaroxaban, however, exhibits its anticoagulation effects independent of AT-III. The aim of the present study was to evaluate the efficacy and safety of rivaroxaban as a treatment for venous thromboembolism in patients with AT-III deficiency due to nephrotic syndrome. Patients with nephrotic syndrome and low serum concentration and functional activity of AT-III and venous thromboembolism were randomly assigned to the rivaroxaban group $(n=8)$ and low weight molecular heparin group $(n=8)$. The patients were treated for 4 weeks and evaluated at weeks 2 and 4 . The primary endpoint was thrombus dissolution or a $>90 \%$ decrease in thrombus volume in 4 weeks. Secondary endpoints included an increase in the volume of the pre-existing thrombosis and safety assessments. In each of the two groups, $7 / 8$ patients achieved a primary endpoint. At week 2, 5 patients in the rivaroxaban group and 4 in the low weight molecular heparin group had achieved the primary endpoint. Notably, at week 2 the patients whose AT-III levels and functional activity remained low in the low weight molecular heparin group did not achieve the primary endpoint. The adverse effects were similar in both groups, with no severe hemorrhage observed. In conclusion, the results of this pilot study demonstrate that rivaroxaban may be an effective, safe, single-agent approach for treating vein thromboembolism in patients with nephrotic syndrome and low AT-III levels. The potential benefits of rivaroxaban over low weight molecular heparin treatment require further investigation with a larger sample size in order to validate the findings of the present study.
\end{abstract}

Correspondence to: Professor Zhihong Liu or Professor Haitao Zhang, National Clinical Research Center of Kidney Disease, Jinling Hospital, Nanjing University School of Medicine, 305 East Zhong Shan Road, Nanjing, Jiangsu 210016, P.R. China

E-mail: liuzhihong@nju.edu.cn

E-mail: htzhang163@163.com

Key words: nephrotic syndrome, venous thromboembolism, antithrombin-III, rivaroxaban, anticoagulation

\section{Introduction}

Venous thromboembolism (VTE), which comprises deep-vein thrombosis (DVT) or pulmonary embolism, is a common disorder with a global annual incidence of 1-2 cases per 1,000 people $(1,2)$. Studies of hospitalized and ambulatory patients have demonstrated that patients with nephrotic syndrome (NS) have an increased risk of 3-50\% of thrombotic events (3-7). Decreased antithrombin (AT)-III is one of the factors contributing to hypercoagulability in patients with NS (3). The risk of VTE in patients with NS and low AT-III is 3 to 7 times higher compared with the general population (8). Standard treatment for VTE is an initial course of heparin, followed by a vitamin K antagonist (VKA) (9). This therapy is effective but requires laboratory monitoring and dose adjustments, which was inconvenient to outpatients. Meanwhile, the anticoagulation of effect of heparin is dependent on adequate circulating AT-III levels (10). AT-III production and consumption constitute a dynamic process. In the presence of reduced circulating levels of AT-III, heparin-mediated thrombin inhibition is deficient, leading to decreased heparin sensitivity (11). This effect is called heparin resistance (HR) or heparin tachyphylaxis $(10,11)$. Reduced AT-III levels due to loss in urine protein are common in patients with NS, with a reported occurrence rate of $86.4 \%$ (12); thus, the anticoagulation efficacy of heparin in these patients is questionable. Rivaroxaban, an oral factor Xa inhibitor, may provide a simple, fixed-dose regimen for treating DVT without laboratory monitoring (13-17). Furthermore, it functions independent of AT-III and may therefore be superior to heparin as a treatment for patients with low AT-III levels. A previous study of AT-III-deficient mice revealed that the clotting time prolongation and antithrombotic effects of oral factor $\mathrm{Xa}$ inhibitors were not affected by AT-III levels, whereas those of AT-dependent anticoagulants (including fondaparinux, enoxaparin, and unfractionated heparin) were attenuated (18). The aim of the present study was to assess the efficacy and safety of rivaroxaban as a treatment for VTE in patients with NS and low AT-III concentration and functional activity as compared with low molecular weight heparin (LMWH) treatment.

\section{Materials and methods}

Patients. Between May 2010 and October 2015, a total of 16 patients were enrolled in the present study; each group had 
8 patients. The patients enrolled in the present study fulfilled the following inclusion criteria: Aged 18-65 year; diagnosed with NS; proteinuria ( $>3.5 \mathrm{~g} / 24 \mathrm{~h})$; serum albumin $(<25 \mathrm{~g} / \mathrm{l})$; serum AT-III concentration $<20 \mathrm{mg} / \mathrm{dl}$ and functional activity $<70 \%$; and a diagnosis of DVT or PE using complete compression ultrasound renal vein or pulmonary artery CT angiography. All patients provide prior informed consent. The primary exclusion criteria were as follows: $\mathrm{Scr} \geq 3 \mathrm{mg} / \mathrm{dl}$; requirement for thrombolytic therapy or inferior vena cava strainer implantation; risk of cerebral ischemia; intracerebral bleeding or gastrointestinal bleeding within the previous 6 months; surgery within the previous 4 weeks; an active peptic ulcer; a known bleeding disorder; prolonged international normalized ratio or activated partial thromboplastin time; platelet count $<100 \times 10^{9}$ cells $/ 1$; treatment with a VKA, unfractionated heparin, LMWH, antiplatelet agents, or potent CYP3A4 inhibitors; impaired liver function (transaminase $>2$-fold above the normal range); poor compliance; and simultaneous enrollment in other clinical trials.

Study design and treatment. The present pilot study is a prospective, active-controlled, open-label, randomized, clinical trial. The present study was approved by the Ethics Committee of Jinling Hospital (Nanjing, China) and adhered to the Declaration of Helsinki and the principles outlined in the 'Guidelines for Good Clinical Practice' at the International Conference on Harmonization Tripartite Guideline (January 1997) (19).

Eligible patients were randomly assigned to the rivaroxaban or LMWH groups. The patients in the rivaroxaban group received $30 \mathrm{mg}$ /day rivaroxaban (Bayer HealthCare Pharmaceuticals LLC, Berlin, Germany) orally and patients in the LMWH group received dalteparin (Pfizer, Inc., New York, NY, USA) $5000 \mathrm{U}$ twice daily via subcutaneous injection. Treatment was discontinued in the two groups at the 2 -week follow up if the thrombus had disappeared and the patients achieved clinical remission.

Outcomes and follow-up. The primary endpoint was dissolution or a $>90 \%$ decrease in thrombus volume in 4 weeks. Secondary endpoints included recurrent VTE, succumbing to thrombosis, volume increase of existing thrombosis. Furthermore, changes of serum AT-III level and safety of anticoagulation treatment were assessed. The principal safety concern was clinically relevant bleeding, defined as a composite of major or clinically relevant non-major bleeding.

Patients were evaluated at the baseline and at weeks 2 and 4. Blood and urine samples were collected for laboratory analysis. Blood coagulation using the CoaguChek XS system (Roche Diagnostics GmbH, Mannheim Germany), AT-III concentration (ACLTOP700; WerfenLife, Barcelona, Spain) and functional activity using the Berichrom ${ }^{\circledR}$ Antithrombin III kit (Siemens Healthineers, Erlangen, Germany), urine protein using biuret colorimetry and clinical chemistry using a 7600 series automatic analyzer (Hitachi, Ltd., Tokyo, Japan) were assessed. Serum AT-III was tested using immune rate turbidimetry and AT-III functional activity was tested via automated chromogenic assay as previously described $(20,21)$.

Complete compression ultrasound, renal vein and pulmonary artery computed tomography (CT) angiography

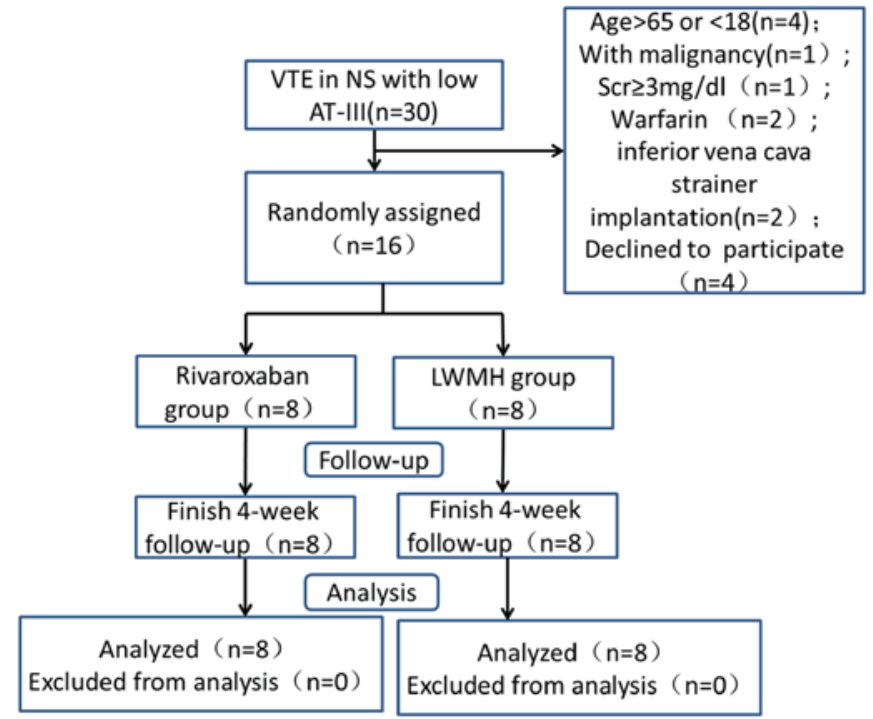

Figure 1. Study flow diagram. VTE, venous thromboembolism; AT-III, antithrombin III; LWMH, low weight molecular heparin.

examinations were performed at the baseline and during each visit. The size of the thrombus was measured using Volume Software of Syngo MMWP (VE32B) workstation of 64-slice dual source CT scanner (Siemens AG, Munich, Germany) and the World Health Organization standard for measuring gross tumor volume was used (22).

Definitions. Low serum AT-III level refers to an AT-III concentration $<20 \mathrm{mg} / \mathrm{dl}$ (23). AT-III deficiency refers to an AT-III functional activity $<70 \%$ (24). Major bleeding is defined as fatal bleeding involving a critical organ or requiring reoperation, or clinically overt bleeding outside the surgical site associated with a decrease in hemoglobin to $\geq 2 \mathrm{~g} / \mathrm{dl}$ or requiring an infusion of $\geq 2$ units of blood. The efficacy of NS was definite complete remission (CR) (proteinuria $<0.4 \mathrm{~g} / 24 \mathrm{~h}$ and serum albumin $>35 \mathrm{~g} / 1$ with normal $\mathrm{SCr}$ ), partial remission $(\mathrm{PR} ; \geq 50 \%$ reduction in proteinuria and urine protein $<3.5 \mathrm{~g} / 24 \mathrm{~h}$, with normal or $\leq 25 \%$ increase in $\mathrm{SCr}$ level from baseline) and no response (NR; not reaching CR or PR definition).

Statistical analysis. Data were analyzed using SPSS 17.0 (SPSS, Inc., Chicago, IL, USA). Normally distributed variables (serum albumin, $24 \mathrm{~h}$ urinary protein, AT-III concentration, AT-III activity, Tchol, triglyceride, Scr, estimated glomerular filtration rate, prothrombin time and activated partial thromboplastin time) were expressed as the mean \pm standard deviation and analyzed using the Student's t-test. Non-parametric variables (age and time of thrombotic events to NS) were expressed as the median values (interquartile range) and compared using the Mann-Whitney U test. Categorical variables were compared using Pearson $\chi^{2}$ test or Fisher's exact test. $\mathrm{P}<0.05$ was considered to indicate a statistically significant difference.

\section{Results}

Study patients. A flow diagram of the study is presented in Fig. 1. There was no family history of thrombosis and all 
Table I. Baseline clinical characteristics of patients in both groups.

\begin{tabular}{|c|c|c|c|}
\hline Characteristic & Rivaroxaban group $(\mathrm{n}=8)$ & LWMH group $(n=8)$ & P-value \\
\hline $\operatorname{Sex}(M / F)$ & $6 / 2$ & $7 / 1$ & 0.5 \\
\hline Age (years) & $35.0(22.3,47.5)$ & $21.0(20.0,43.8)$ & 0.579 \\
\hline \multicolumn{4}{|l|}{ Definite causes of renal disease } \\
\hline MCD & 5 & 2 & \\
\hline $\mathrm{MN}$ & 2 & 2 & \\
\hline FSGS & 0 & 2 & \\
\hline $\mathrm{LN}$ & 0 & 1 & \\
\hline Unknown & 1 & 1 & \\
\hline \multicolumn{4}{|l|}{ Treatment for renal disease } \\
\hline Pred & 4 & 4 & \\
\hline Pred+CTX & 2 & 2 & \\
\hline Pred+TW & 2 & 1 & \\
\hline Pred+CsA & 0 & 1 & \\
\hline Time of thrombotic events to NS onset (m) & $0.58(0.25,1.18)$ & $0.50(0.31,2.38)$ & 0.402 \\
\hline Serum albumin $(\mathrm{g} / \mathrm{l})$ & $18.76 \pm 1.90$ & $20.07 \pm 3.56$ & 0.374 \\
\hline $24 \mathrm{~h}$ urinary protein $(\mathrm{g} / 24 \mathrm{~h})$ & $13.36 \pm 5.9$ & $8.62 \pm 3.13$ & 0.065 \\
\hline AT-III concentration (mg/dl) & $14.6 \pm 4.8$ & $16.7 \pm 3.6$ & 0.337 \\
\hline AT-III functional Activity (\%) & $43.6 \pm 21.5$ & $48.6 \pm 17.7$ & 0.408 \\
\hline Tchol (mmol/l) & $11.53 \pm 2.18$ & $12.37 \pm 1.7$ & 0.978 \\
\hline TG (mmol/l) & $3.45 \pm 1.98$ & $3.47 \pm 1.37$ & 0.507 \\
\hline $\mathrm{SCr}(\mathrm{mg} / \mathrm{dl})$ & $1.066 \pm 0.47$ & $0.95 \pm 0.15$ & 0.598 \\
\hline eGFR $\left[\mathrm{ml} /\left(\min .1 .73 \mathrm{~m}^{2}\right)\right]$ & $99.02 \pm 31.45$ & $106.64 \pm 24.65$ & 0.863 \\
\hline PT (sec) & $11.05 \pm 1.13$ & $11.15 \pm 1.14$ & 0.627 \\
\hline APTT (sec) & $35.5 \pm 5.6$ & $33.9 \pm 4.4$ & 0.521 \\
\hline Fibrin (mg/dl) & $481 \pm 49$ & $532 \pm 121$ & 0.288 \\
\hline
\end{tabular}

The data are expressed as the median values (25th, 75 th percentiles) or the mean values (SD). The reference ranges are as follows: $24 \mathrm{~h}$ urinary protein, $\leq 0.4$ g/d; Alb, 35-55 g/l; SCr, 0.51-1.24 mg/dl; Serum AT-III, 25-36 mg/dl; Tchol, 3-6 mmol/l; TG, 0.28-2.2 mmol/1; PT, 10.5-13.0 S; APTT, 25.0-40.0 S; Fibrin, 200-400 mg/dl. M, male; F, female; MCD, minimal change disease; MN, membranous nephropathy; FSGS, focal segmental glomerulosclerosis; LN, lupus nephritis; Pred, prednisone; CTX, Cytoxan; CsA, cyclosporin A; TW, tripterygium wilfordii; Alb, albumin; Tchol, cholesterol; TG, triglyceride; Scr, serum creatinine; eGFR, estimated glomerular filtration rate; PT, prothrombin time; APTT, activated partial thromboplastin time.

patients suffered from acute VTEs. No significant differences were observed in the baseline characteristics of the groups (Table I).

Distribution of thrombosis. Thrombosis distribution is presented in Table II. Thrombus occurred in 3 sites for 3 patients, 2 sites for 1 patient and 1 site for 4 patients in the rivaroxaban group. In the LWMH group, thrombus presented in 3 sites for 1 patient, 2 sites for 3 patients, and 1 site for 4 patients. A total of 5 patients in the rivaroxaban group and 6 patients in the LWMH group had DVT.

Primary endpoint. The primary endpoint occurred in 7 patients from the rivaroxaban group and 7 from the LWMH group at week 4. At week 2, 5 patients in the rivaroxaban group and 4 patients in the LWMH group achieved the primary endpoint. Of the patients with DVT, 4 in the rivaroxaban group and 4 in the LWMH group achieved the primary endpoint at week 4 (Fig. 2). A total of 8 patients with PE in the rivaroxaban group
Table II. Distribution of thrombosis in the rivaroxaban and LWMH groups.

\begin{tabular}{lcc}
\hline $\begin{array}{l}\text { Position } \\
\text { of thrombus }\end{array}$ & $\begin{array}{c}\text { Rivaroxaban } \\
\text { group }(\mathrm{n}=8)\end{array}$ & $\begin{array}{c}\text { LWMH } \\
\text { group }(\mathrm{n}=8)\end{array}$ \\
\hline Renal vein & 4 & 6 \\
Bilateral renal vein & 1 & 0 \\
Left renal vein & 2 & 4 \\
Right renal vein & 1 & 2 \\
Inferior cava vein & 3 & 1 \\
Pulmonary artery & 8 & 6
\end{tabular}

LWMH, low weight molecular heparin.

achieved the primary endpoint at week 4, whereas 5 of 6 in the LWMH group achieved the primary endpoint (Fig. 3). 


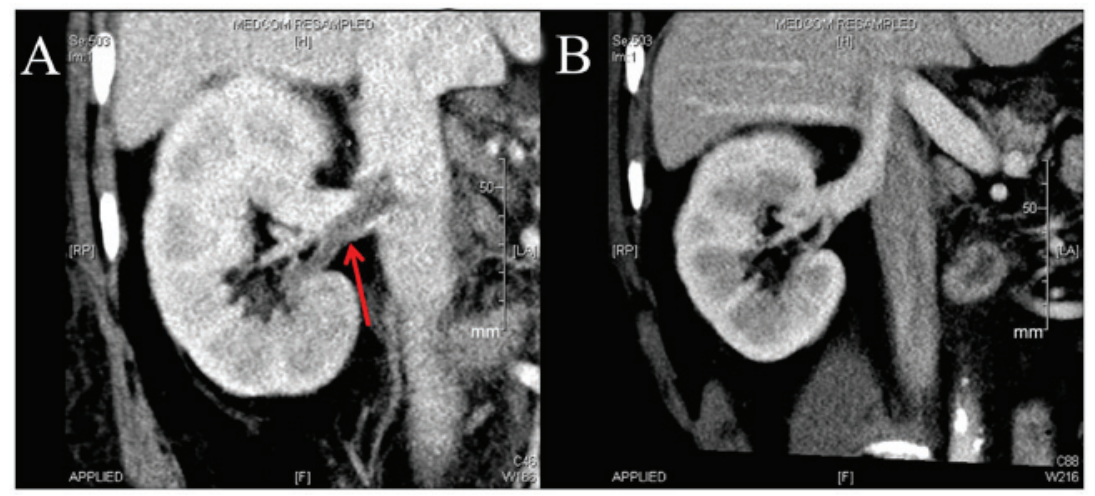

Figure 2. Changes in deep vein thrombosis following LWMH treatment of one patient. (A) CT angiography at baseline. Right renal vein thrombosis is indicated by the red arrow. (B) CT angiography at week 2 . Right renal vein thrombosis was no longer visible.

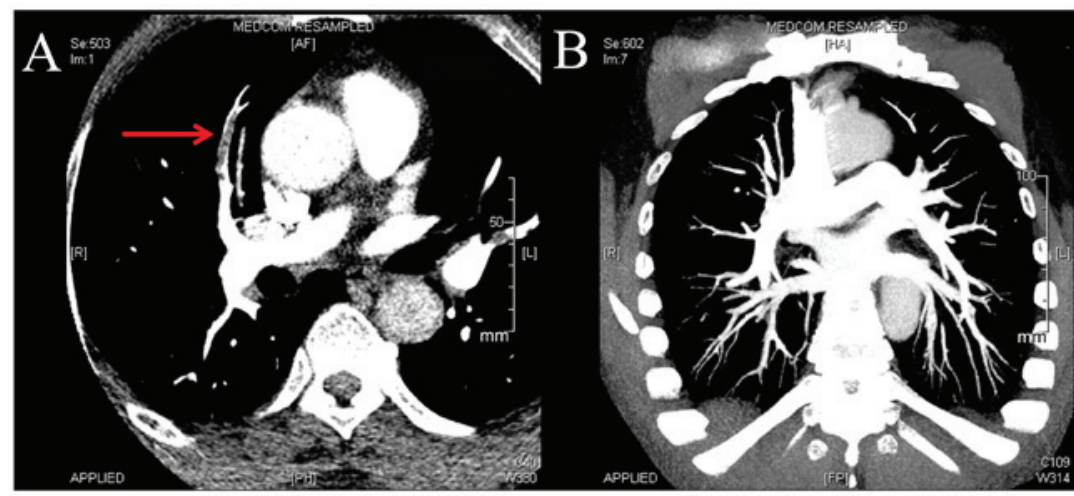

Figure 3. Changes in PE after rivaroxaban treatment of one patient. (A) CT angiography at baseline. Right PE is indicated by the red arrow. (B) CT angiography at week 2. Right PE was no longer visible. PE, pulmonary embolism.
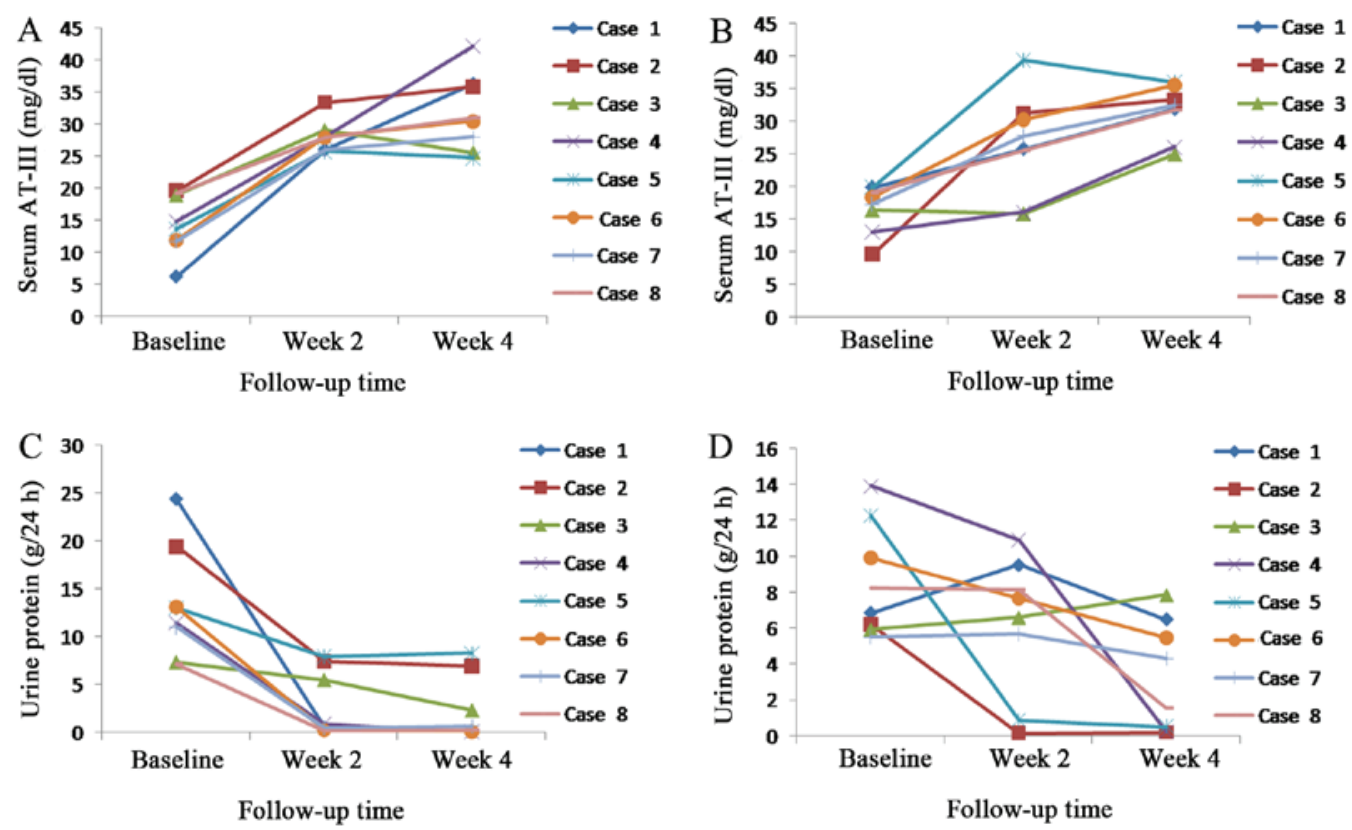

Figure 4. Changes in serum AT-III concentration and urine protein. Serum AT-III concentration in the (A) rivaroxaban and (B) LWMH groups. Urine protein in the (C) rivaroxaban group and (D) LWMH groups. AT-III, antithrombin III; LWMH, low weight molecular heparin.

Serum AT-III changes and efficacy of NS. Urine protein decreased and serum AT-III concentration rose with increased treatment time in the rivaroxaban and LWMH groups (Fig. 4).
Serum AT-III concentration and functional activity returned to a normal level at week 2 for all patients in the rivaroxaban group, whereas in the LWMH group serum levels of AT-III 
returned to normal at week 2 for 6 patients at week 4 for 2 patients. A total of 5 patients achieved remission (PR, 3;CR, 2) in the rivaroxaban group, as did 4 patients (PR, 2; CR, 2) in the LWMH group at week 4 . The 2 patients in the LWMH group whose AT-III concentration and functional activity remained low at week 2 did not achieve the primary endpoint.

Adverse events. No major bleeding occurred in any group. Skin ecchymosis occurred in 1 patient in the rivaroxaban group and 3 in the LWMH group. Submucosal hemorrhage occurred in the oral cavity in 1 patient in the rivaroxaban group. No patient in either group had the combination of an alanine aminotransferase level $>3 x$ the upper limit of the normal range or a bilirubin level $>2 \mathrm{x}$ the upper limit of the normal range.

\section{Discussion}

This present study demonstrated that rivaroxaban has similar efficacy and safety as LWMH when used as a treatment for VTE in patients with NS and low AT-III levels. No significant differences in the primary endpoint and the occurrence of adverse events were observed between the treatment groups.

Theoretically, as a direct Xa inhibitor and AT-III independent agent, rivaroxaban should be superior to LWMH in patients with NS and low AT-III levels. The results of the present study, however, differ, which may be because the serum AT-III concentration and functional activity returned to normal following therapy, enhancing the efficacy of LWMH. The serum AT-III concentration and functional activity returned to normal at week 2 in all patients treated with rivaroxaban and in 6 patients in the LWMH group. The recovery of AT-III levels in both groups may be attributed to the following factors: i) Patients achieved remission in NS after therapy, which reduced the loss of AT-III from the urine; ii) corticosteroids may elevate the serum AT-III levels by promoting its synthesis (25).

The results of the present study demonstrate that the anticoagulation efficacy during the first weeks of treatment was similar for rivaroxaban and LWMH treatment. However, the number of patients achieving the primary endpoint was slightly higher in the rivaroxaban group at week 2 . This observation suggests that rivaroxaban was more effective than LWMH for the treatment of VTE in patients with persistently low serum AT-III levels. Additionally, at week 2, the two patients in the LWMH group with low serum AT-III levels did not achieve the primary endpoint, suggesting that the anticoagulation effect of LWMH decreased with low serum AT-III levels. Thus, rivaroxaban may be suitable for the treatment and prevention of VTE in patients with NS and low AT-III levels.

In the present study, the tome from occurrence of thrombotic events to NS onset ranged from 0.25 to 2.38 months. Bellomo and Atkins (4) reported that $76.2 \%$ of thrombotic events occurred in the first 6 months following the initial presentation of NS. Mahmoodi et al (6) reported that the incidence of VTE was $9.85 \%$ when calculated over the first 6 months of observation, a 9.7-fold increase over the mean annual incidence, with 0.9 years as the median observation period until the first VTE was observed. Li et al (7) reported that, in patients with membranous nephropathy, venous thrombotic events occurred in the first months (mean, 3.5 months) following the initial presentation of NS. The risk of thrombotic events is highest in the first months following NS diagnosis and the majority of experts recommend that prophylactic anticoagulation be performed in the first 6 months (6).

The present study has several limitations that should be noted. Firstly, this was a prospective exploratory study with a small sample size of 16 patients. Secondly, the serum AT-III level in the majority of patients increased to normal during treatment; therefore, AT-III was not persistently low. Thirdly urine AT-III levels were not tested to verify that AT-III deficiency was due to NS. However, AT-III function and levels were low in all patients at the baseline and AT-III levels recovered over time, which suggests acquired AT deficiency as opposed to inherited AT deficiency. Acquired AT-III deficiency occurs as a result of either decreased production (due to factors including liver impairment), increased consumption [due to factors including sepsis and diffuse intravascular coagulation (DIC)] or increased renal loss, as in NS. No serious liver impairment, sepsis or DIC, was observed in patients the present study, which suggests that NS was the cause of low AT-III levels and functionality.

In conclusion, as an oral direct factor $\mathrm{Xa}$ inhibitor, rivaroxaban has superior effects compared with traditional anticoagulants, functions independently of serum AT-III levels, has good compliance and does not require monitoring. The results of the present study demonstrate that rivaroxaban $(30 \mathrm{mg} /$ day for 4 weeks) may provide an effective, safe, single-agent approach for treating VTE in patients with NS and low AT-III levels. Future research is required to explore rivaroxaban's anticoagulation effect for treating VTE in patients with refractory NS whose AT-III levels are persistently low.

\section{Acknowledgements}

The present study was supported by the Primary Funding Source: National Key Technology R\&D Program (grant nos. 2013BAI09B04 and 2015BAI12B05) and the Clinical Medical Research Center Project of Jiangsu province (grant no. BL2012007).

\section{References}

1. Oger E: Incidence of venous thromboembolism: A community-based study in Western France. EPI-GETBP study group. Groupe d'Etude de la thrombose de bretagne occidentale. Thromb Haemost 83: 657-660, 2000.

2. Spencer FA, Emery C, Lessard D, Anderson F, Emani S, Aragam J, Becker RC and Goldberg RJ: The worcester venous thromboembolism study: A population-based study of the clinical epidemiology of venous thromboembolism. J Gen Intern Med 21: 722-727, 2006

3. Llach F: Hypercoagulability, renal vein thrombosis and other thrombotic complications of nephrotic syndrome. Kidney Int 28 : 429-439, 1985.

4. Bellomo R and Atkins RC: Membranous nephropathy and thromboembolism: Is prophylactic anticoagulation warranted? Nephron 63: 249-254, 1993.

5. Barbour SH, Greenwald A, Djurdjev O, Levin A, Hladunewich MA, Nachman PH, Hogan SL, Cattran DC and Reich HN: Disease-specific risk of venous thromboembolic events is increased in idiopathic glomerulonephritis. Kidney Int 81: 190-195, 2012. 
6. Mahmoodi BK, ten Kate MK, Waanders F, Veeger NJ, Brouwer JL, Vogt L, Navis G and van der Meer J: High absolute risks and predictors of venous and arterial thromboembolic events in patients with nephrotic syndrome: Results from a large retrospective cohort study. Circulation 117: 224-230, 2008.

7. Li SJ, Guo JZ, Zuo K, Zhang J, Wu Y, Zhou CS, Lu GM and Liu ZH: Thromboembolic complications in membranous nephropathy patients with nephrotic syndrome-a prospective study. Thromb Res 130: 501-505, 2012.

8. Kauffman RH, Velgkamp JJ, Van Tilburg NH and Van Es LA: Acuqired antithrombin III defiency and thrombosis in the nephrotic syndrome. Am J Med 65: 607-613, 1978.

9. Büller HR, Agnelli GA, Hull RD, Hyers TM, Prins MH and Raskob GE: Antithrombotic therapy for venous thromboembolic disease: The seventh ACCP conference on antithrombotic and thrombolytic therapy. Chest 126 (Suppl 3): 401S-428S, 2004.

10. Bharadwaj J, Jayaraman C and Shrivastava R: Heparin resistance. Lab Hematol 9: 125-131, 2003.

11. Spiess BD: Treating heparin resistance with antithrombin or fresh frozen plasma. Ann Thorac Surg 85: 2153-2160, 2008.

12. Saxena R, Batra VV and Singh ND: Prothrombotic factors in nephritic syndrome. Indian J Pathol Microbiol 43 : 319-323, 2000

13. Kubitza D, Becka M, Voith B, Zuehlsdorf M and WenSing G: Safety, pharmacodynamics, and pharmacokinetics of single doses of BAY 59-7939, an oral, direct factor Xa inhibitor. Clin Pharmacol Ther 78: 412-421, 2005.

14. Eriksson BI, Kakkar AK, Turpie AG, Gent M, Bandel TJ, Homering M, Misselwitz F and Lassen MR: Oral rivaroxaban for the prevention of symptomatic venous thromboembolism after elective hip and knee replacement. J Bone Joint Surg Br 91 : 636-644, 2009

15. Turpie AG, Lassen MR, Davidson BL, Bauer KA, Gent M, Kwong LM, Cushner FD, Lotke PA, Berkowitz SD, Bandel TJ, et al: Rivaroxaban versus enoxaparin for thromboprophylaxis after total knee arthroplasty (RECORD4): A randomised trial. Lancet 373: 1673-1680, 2009.

16. EINSTEIN Investigators; Bauersachs R, Berkowitz SD, Brenner B, Buller HR, Decousus H, Gallus AS, Lensing AW, Misselwitz F, Prins MH, et al: Oral rivaroxaban for symptomatic venous thromboembolism. N Engl J Med 363: 2499-2510, 2010.
17. EINSTEIN-PE Investigators; Büller HR, Prins $\mathrm{MH}$, Lensin AW, Decousus H, Jacobson BF, Minar E, Chlumsky J, Verhamme P, Wells $\mathrm{P}$, et al: Oral rivaroxaban for the treatment of symptomatic pulmonary embolism. N Engl J Med 366: 1287-1297, 2012

18. Fukuda T, Kamisato C, Honda Y, Matsushita T, Kojima T, Furugohri T, Morishima Y and Shibano T: Impact of antithrombin deficiency on efficacy of edoxaban and antithrombin-dependent anticoagulants, fondaparinux, enoxaparin, and heparin. Thromb Res 131: 540-546, 2013

19. Switula D: Principles of good clinical practice (GCP) in clinical research. Sci Eng Ethics 6: 71-77, 2000.

20. Antovic J, Söderström J, Karlman B and Blombäck M: Evaluation of a new immunoturbidimetric test (LIATEST Antithrombin III) for determination of antithrombin antigen. Clin Lab Haem 23: 313-316, 2001.

21. Ungerstedt JS, Antovic J, Blombäck M, Bremme K and Johnsson $\mathrm{H}$ : Antithrombin antigen and activity in patients with acquired antithrombin deficiency-is there a difference? J Thromb Haemost 2: 838-839, 2004.

22. Ak G, Metintas M, Metintas S, Yildirim H, Ozkan R and Ozden H: Three-dimensional evaluation of chemotherapy response in malignant pleural mesothelioma. Eur J Radiol 74: 130-135, 2010.

23. Tang $X$, Wang $X F$, Zhang $L H$, Chen $Z H$, Zhang J, Zhang Y, Zeng $\mathrm{CH}$ and Liu ZH: The assay and clinical significance of plasma antithrombin III in adult nephritic syndrome patients. J Nephrol Dialy Transplant 19: 407-411, 2010.

24. Ranucci M: Antithrombin III. Key factor in extracorporeal circulation. Minerva Anestesiol 68: 454-457, 2002.

25. Meade TW, Dyer S, Howarth DJ, Imeson JD and Stirling Y: Antithrombin III and procoagulant activity: Sex differences and effects of the menopause. Br J Haematol 74: 77-81, 1990.

This work is licensed under a Creative Commons Attribution-NonCommercial-NoDerivatives 4.0 International (CC BY-NC-ND 4.0) License. 